\title{
Economic Value of Pharmacogenetic Testing for Cancer Drugs with Clinically Relevant Drug-Gene Associations: A Systematic Literature Review
}

\author{
Fahim Faruque, PharmD; Heejung Noh, PharmD; Arif Hussain, MD; Edward Neuberger, PharmD, MBA; \\ and Eberechukwu Onukwugha, MS, PhD
}

\begin{abstract}
BACKGROUND: Pharmacogenetic testing can provide predictive insights about the efficacy and safety of drugs used in cancer treatment. Although many drug-gene associations have been reported in the literature, the strength of evidence supporting each association can vary significantly. Even among the subgroup of drugs classified by the PharmGKB database to have a high or moderate level of evidence, there is limited information regarding the economic value of pharmacogenetic testing.
\end{abstract}

OBJECTIVES: To: (a) summarize the available pharmacoeconomic evidence assessing the value of pharmacogenetic testing for cancer drugs with clinically relevant drug-gene associations; (b) determine the quality of the studies that contain this evidence; and (c) discuss the quality of this evidence with respect to the level of evidence of the drug-gene associations.

METHODS: The PharmGKB database was used to identify cancer drugs with clinically relevant drug-gene associations graded high $(1 \mathrm{~A}, 1 \mathrm{~B})$ or moderate $(2 \mathrm{~A}, 2 \mathrm{~B})$. A systematic literature review was conducted using these drugs. Ovid MEDLINE and Embase databases were searched to identify costeffectiveness, cost-utility, or cost-minimization studies comparing pharmacogenetic testing to an alternative. Cost and effect values from every relevant comparison within the studies were extracted, and the incremental cost-effectiveness ratio (ICER) was either extracted or calculated for each comparison. Quality assessment was conducted for each study using the Quality of Health Economic Studies (QHES) instrument. Qualitative synthesis was used to summarize the data.

RESULTS: The search yielded 2,191 citations, of which 35 studies met the inclusion criteria. Pharmacoeconomic studies were available for the following drugs from the PharmGKB database: fluoropyrimidine, 6-mercaptopurine, irinotecan, carboplatin, cisplatin, erlotinib, gefitinib, cetuximab, panitumumab, and trastuzumab. The studies were conducted in Asia, Europe, Canada, the United States, and Mexico and reported cost-utility, cost-effectiveness, and cost-minimization outcomes. The mean QHES score was $80(S D=22)$ for the studies of drug-gene pairs with high ( $1 A$, $1 B$ ) and moderate $(2 A, 2 B)$ levels of evidence $(1 A=82,1 B=93,2 A=71$, and $2 B=74)$. There was variation across studies in terms of reporting. 109 relevant comparisons were identified within the studies. Of those that reported cost per life-year or cost per quality-adjusted life-year $(n=58$ comparisons), pharmacogenetic testing was dominant in $21 \%$ overall and $42 \%, 21 \%, 17 \%$, and $5 \%$ of the comparisons in Asia, Europe, Canada, and the United States, respectively. Variability was observed in the ICER values regardless of geographic region or drug. Pharmacogenetic testing was cost saving in 17 of 19 cost-minimization comparisons and was favored most frequently when compared with genetically indiscriminate strategies containing the drug of interest.

CONCLUSIONS: There was mixed evidence regarding the value of pharmacogenetic testing to guide cancer treatment. For future pharmacogenomicrelated economic studies, we recommend prioritizing clinically relevant drug-gene associations and greater adherence to available best practice guidelines for conducting and reporting economic evaluation studies.

J Manag Care Spec Pharm. 2019;25(2):260-71

Copyright $\odot 2019$, Academy of Managed Care Pharmacy. All rights reserved.

\section{What is already known about this subject}

Pharmacogenetics enables health care providers to more appropriately select treatments based on a patient's genes. Clinical research comparing drug and gene pairs demonstrates that the degree of association with either efficacy or safety outcomes varies based on the pairs selected.

- Precision medicine generally shows favorable economic outcomes when the aggregate of all therapeutic areas is considered.

\section{What this study adds}

This systematic literature review summarizes the incremental cost-effectiveness ratios (ICERs) of pharmacogenetic testing for cancer drugs with clinically relevant drug-gene associations based on currently published cost-effectiveness, cost-utility, and cost-minimization studies.

- Since the body of the reviewed published literature was assessed at a comparison level rather than a study level, this review identifies and accounts for all ICER values from the evaluated studies, including those that the authors did not explicitly summarize, thus providing additional insights into the economics of pharmacogenetic testing in cancer.

- This study underscores the absence of methodologic consistency among the various published studies on the economics of pharmacogenetic testing, and it also provides suggestions for more consistent alignment among future studies in this area.

$\mathrm{P}$ harmacogenetics, "the study of variations in DNA sequence as related to drug response," is at the forefront of precision medicine (i.e., personalized medicine). ${ }^{1}$ Precision medicine enables health care providers to use the knowledge of biological variations that inherently exist within populations to individualize care for patients. Thus, it is especially relevant in oncology, where heterogeneity among cancer 


\section{Economic Value of Pharmacogenetic Testing for Cancer Drugs with Clinically Relevant Drug-Gene Associations: A Systematic Literature Review}

types creates a need to develop targeted therapies for biologically unique populations. ${ }^{2,3}$

The PharmGKB database contains a collection of clinically relevant drug-gene associations developed using an evidencebased approach, and the PharmGKB group systematically grades the level of evidence that supports such associations as high (1A, 1B), moderate (2A, 2B), low, and preliminary. The associations are drug-gene relationships with respect to dosage, efficacy, toxicity/adverse drug reactions, and metabolism/pharmacokinetics. ${ }^{4}$ While there is an evidence basis to identify clinically promising drug-gene associations, there is limited information available regarding their economic value. This insight is essential in oncology because the cancer treatment paradigm is shifting toward individualized therapy. ${ }^{5}$ The shift is also likely to affect the economics of cancer care. For instance, does a drug provide relatively more economic value when prescribed for cancers based on a specific biomarker or when prescribed based on tumor origin and tumor histology? $?^{6,7}$ The former has the potential to be a more tissue-agnostic approach.

To our knowledge, only 1 systematic literature review (SLR) has attempted to understand the economic value from a druggene relevance perspective. ${ }^{8}$ Furthermore, no SLRs have been conducted to understand the economic value of using predictive pharmacogenetic tests of cancer drugs. To address this gap in the literature, we conducted an SLR to (a) summarize the available evidence assessing the economic value of pharmacogenetic testing for cancer therapies with clinically relevant drug-gene associations; (b) determine the quality of the aforementioned studies; and (c) discuss the quality of the evidence assessing the economic value of pharmacogenetic testing with respect to the level of evidence of the drug-gene pair association as determined by PharmGKB. ${ }^{4}$

\section{Methods}

An SLR was conducted based on work from a previous literature review presented as a poster and reported according to PRISMA guidelines. ${ }^{910}$ The previous review reported the number and quality of pharmacoeconomic studies on genetic testing for selected agents from PharmGKB, comparison of the economic evidence, and regulatory agency recommendations for genetic testing with the drug-gene pair level of evidence. ${ }^{9}$ The study protocol was registered with PROSPERO, an international database of prospectively registered systematic reviews. ${ }^{11}$ The Embase and MEDLINE databases were searched for literature published between January 1, 2000, and September 15, 2016. The search strategy was composed of terms for genetics, economics, and drug names, and the 3 categories were combined with the "AND" Boolean operator. The search was restricted to studies with an available abstract and peer-reviewed full report, conducted in human subjects, and reported in English. The full search strategy is reported in the Appendix (available in online article).

\section{Study Selection}

Two reviewers independently screened the titles and abstracts using the inclusion criteria established before the start of the study. Both reviewers independently assessed full texts retrieved for potential inclusion. Studies identified in the previous literature review that met the inclusion criteria were also reviewed independently for inclusion. All disagreements were resolved by discussion between reviewers. Studies were included if they met the following criteria: (a) were an original research article; (b) assessed a population with a cancer in which pharmacogenetic testing affects drug utilization outcomes; (c) assessed cancer drugs for which the level of evidence supporting the drug-gene pair association is $1 \mathrm{~A}, 1 \mathrm{~B}, 2 \mathrm{~A}$, or $2 \mathrm{~B}$ as determined by PharmGKB Clinical Annotations (see the Appendix for drug list and explanation of how drugs were selected); (d) used pharmacogenetic testing to guide therapy compared with no testing, standard care, or any alternative methods; and (e) reported outcomes of cost-effectiveness, cost-utility, or cost-minimization analyses. Editorials, letters, historical articles, commentaries, meta-analyses, reviews, and studies reporting cost of illness, budget impact, or clinical outcomes only were excluded.

\section{Data Extraction}

Data extraction was conducted by 2 authors for each article and the items presented in this report include the following: drug, gene, gene prevalence, disease, country, costing perspective, time horizon, analytic approach, currency, test cost, treatment strategies compared, treatment strategy effectiveness, treatment strategy cost, and economic outcome of comparison (base-case and majority population value reported only when studies reported multiple cost/effect and incremental cost-effectiveness ratio [ICER] values).

\section{Quality Assessment}

Quality assessment was conducted for each study by 2 authors using the Quality of Health Economic Studies (QHES) instrument, and the intraclass correlation coefficient (ICC) was calculated to assess inter-rater reliability using inputs from 2-factor analysis of variance without replication. The QHES instrument is validated to assess the quality of cost-effectiveness studies. It provides a quantitative overall score for each study on a scale ranging from 0 (lowest quality) to 100 (highest quality). ${ }^{12}$ We prespecified each criterion of this tool using a previously published study that also utilized this instrument. ${ }^{13}$ Journals in which included studies were published were characterized as "economic/policy" or "clinical/other" based on their InCites Journal Citation Reports descriptions.

\section{Findings Summary}

Because of the heterogeneity in the methods, outcomes, and settings of included studies, it was inappropriate to conduct a meta-analysis. 


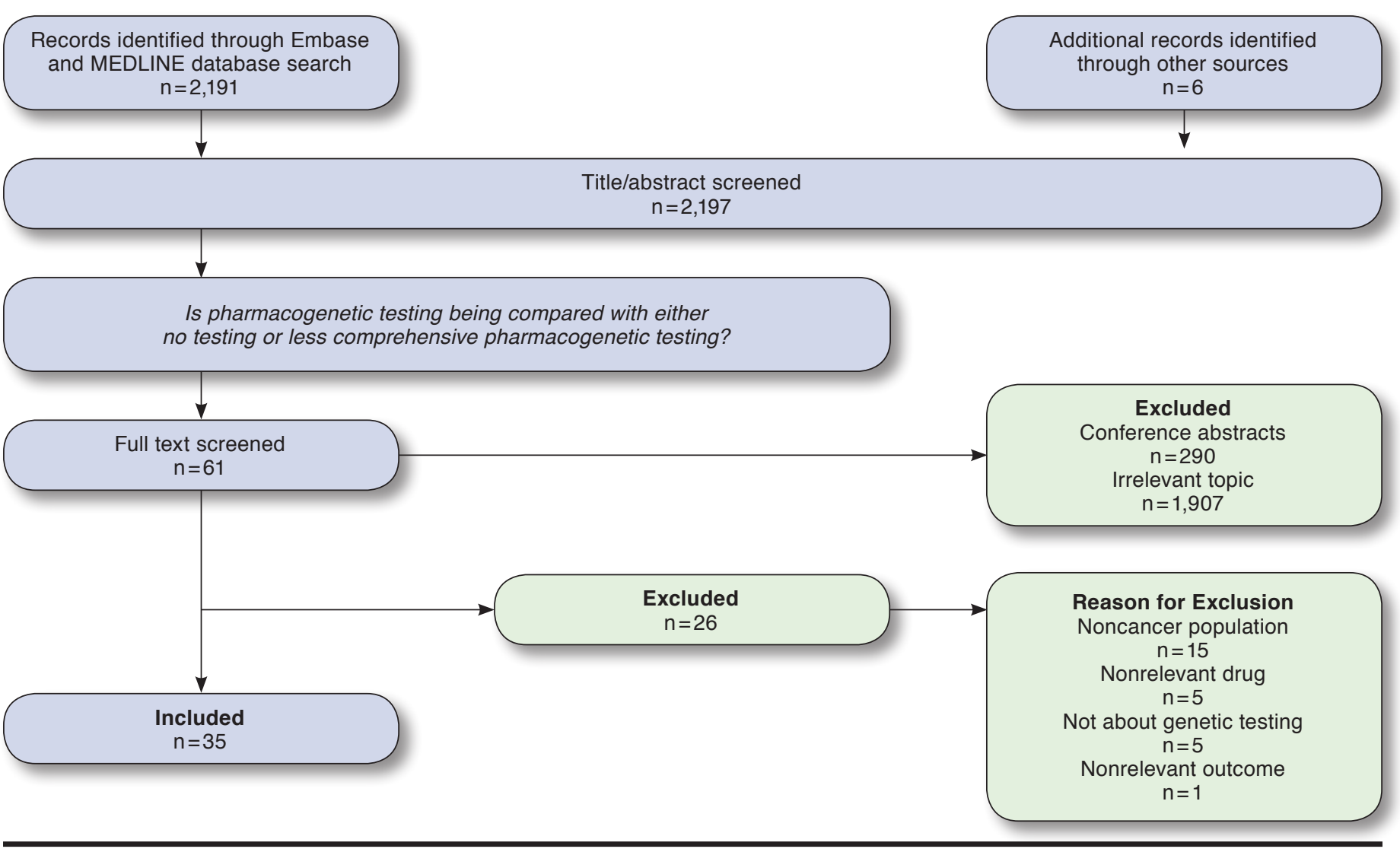

The findings of this study are reported as a qualitative synthesis of economic outcomes at a comparison level. Unlike a synthesis that is conducted at a study level, where each study contributes 1 ICER to the synthesis, a comparison-level synthesis enables 1 study to contribute multiple ICERs because multiple comparisons can be made among the treatment strategies included in the study. In this comparison-level qualitative synthesis of economic outcomes, all comparisons identified within the studies for a drug are weighed equally in the summary of its economic value. Economic outcomes of interest, which include ICERs and incremental cost-utility ratios (both referred as "ICER" in this study), and cost savings or increases were calculated for relevant comparisons using the extracted treatment strategy effect/cost values in situations where the studies did not report a value or reported "extended dominance." For example, Behl et al. (2012) included 4 treatment strategies in their study assessing the economic value of genetic testing for cetuximab. The authors do not report the ICER for the comparison between KRAS testing and no testing. ${ }^{14}$ In this study, we used the cost and effectiveness values provided by the authors for each treatment strategy to calculate the ICER of KRAS testing compared with no testing. Economic results were categorized based on willingness-to-pay (WTP) thresholds reported in the literature. ${ }^{15-17}$ The results were summarized with respect to geographic region, drug, and treatment strategies compared. Quality assessments of studies were summarized by drug and journal category, and we report the average of the scores determined by both authors. Level of evidence for drug-gene pairs, as determined by PharmGKB, was also compared with the quality assessment scores.

\section{Results}

\section{Search Results}

The Embase and MEDLINE database search produced 2,191 abstracts. Six additional abstracts were identified from other sources. Title/abstract screening yielded 61 articles for fulltext review, of which 35 met the inclusion criteria (Figure 1). Twenty-five percent of the drugs identified via the Clinical Annotations list in PharmGKB had at least 1 article included in this study. 


\section{Economic Value of Pharmacogenetic Testing for Cancer Drugs with Clinically}

Relevant Drug-Gene Associations: A Systematic Literature Review

\begin{tabular}{|c|c|c|}
\hline Category & Subcategory & $\begin{array}{r}\text { Number of } \\
\text { Articles (\%) }\end{array}$ \\
\hline \multirow{4}{*}{$\begin{array}{l}\text { Analytic approach } \\
\text { (study model) }\end{array}$} & Simulation modela & $3(9)$ \\
\hline & Markov model & $16 \quad(46)$ \\
\hline & Decision tree & $9 \quad(26)$ \\
\hline & Not reported & $7 \quad(20)$ \\
\hline \multirow[t]{5}{*}{ Perspective } & Private payer & $11 \quad(31)$ \\
\hline & Government payer & $13 \quad(37)$ \\
\hline & Societal & $6 \quad(17)$ \\
\hline & Institution/provider & $3(9)$ \\
\hline & Not reported & $2(6)$ \\
\hline \multirow[t]{6}{*}{ Time horizon } & $<1$ year & $2(6)$ \\
\hline & 1 to $<5$ years & $6 \quad(17)$ \\
\hline & 5 to $<10$ years & $2(6)$ \\
\hline & $\geq 10$ years & $6 \quad(17)$ \\
\hline & Lifetime & $11 \quad(31)$ \\
\hline & Not reported & $8 \quad(23)$ \\
\hline \multirow[t]{5}{*}{ Geographic region } & United States $^{\mathrm{b}}$ & $8 \quad(23)$ \\
\hline & Europe & $12(34)$ \\
\hline & Asia & $9 \quad(26)$ \\
\hline & Canada & $5 \quad(14)$ \\
\hline & Mexico & 1 (3) \\
\hline
\end{tabular}

${ }^{a}$ Discrete event simulation, MonteCarlo simulation model, and microsimulation. ${ }^{b}$ One article also studied Germany; total studies, $n=35$.

\section{Study Characteristics}

Study characteristics are summarized in Table 1 by analytic approach, perspective, time horizon, and geographic region. Eighty-one percent of the studies were published between 2010 and 2016. Respectively, 46\%, 26\%, and 9\% of studies reported Markov models, decision trees, and simulation models as the analytical approach (i.e., study model). Twenty percent did not report an analytical approach. Studies most frequently reported the government payer and private payer perspectives (37\% and $31 \%$ of studies, respectively), followed by societal (17\%) and institution/provider (9\%). Six percent of studies did not report any perspective. Time horizon ranged from $<1$ year to lifetime and was not reported in 8 studies. Studies were conducted most frequently in Europe (public and/or private payers), followed by Asia (public and/or private payers), the United States (private payer), Canada (public payer), and Mexico (public and private payer) and included cost-utility, cost-effectiveness, and cost-minimization analyses. ${ }^{18,19}$

\section{Quality Assessment}

The quality assessment results are summarized in Table 2 . The ICC was determined to be 0.82 (95\% confidence interval $[C I]=0.46-0.93)$. Seventeen percent of the studies were published in economic/policy journals; these studies had higher QHES scores than those published in clinical/other journals (mean $=84$, range $=58-99$ vs. mean $=79$, range $=21-99$ ). Overall, the mean QHES score was 80 (standard deviation=22).
TABLE 2 Study Quality with Respect to Drugs and Journal Category

\begin{tabular}{c|c|c|c}
\hline & $\begin{array}{c}\text { Number } \\
\text { of } \\
\text { Articles }\end{array}$ & $\begin{array}{c}\text { QHES Score } \\
\text { Mean } \\
\text { (Range) }\end{array}$ & $\begin{array}{c}\text { Drug-Gene } \\
\text { Association } \\
\text { Strength }\end{array}$ \\
\hline
\end{tabular}

\begin{tabular}{|c|c|c|c|c|}
\hline \multicolumn{5}{|l|}{ Drug/gene } \\
\hline 5-FU; capecitabine/DPYD & 1 & & 86 & $1 \mathrm{~A}$ \\
\hline 6-mercaptopurine/TPMT & 2 & 78 & $(70-87)$ & $1 \mathrm{~A}$ \\
\hline Erlotinib/EGFR & 6 & 96 & $(89-99)$ & $1 \mathrm{~B}$ \\
\hline Gefitinib/EGFR & 6 & 89 & $(83-95)$ & $1 \mathrm{~B}$ \\
\hline Irinotecan/UGT1Al & 4 & 71 & $(25-98)$ & $2 \mathrm{~A}$ \\
\hline Cetuximab/RAS; KRAS, BRAF & 11 & 71 & $(20-96)$ & $2 \mathrm{~A}$ \\
\hline Panitumumab/KRAS & 2 & 76 & $(72-80)$ & $2 B$ \\
\hline Cisplatin/TPMT; COMT & 1 & & 58 & - \\
\hline Trastuzumab; HER-2 status & 3 & 78 & $(58-89)$ & - \\
\hline $\begin{array}{l}\text { Carboplatin, cyclophosphamide/ } \\
\text { BRCA-1-like }\end{array}$ & 1 & & 96 & - \\
\hline \multicolumn{5}{|l|}{ Journal category } \\
\hline Economic/policy & 6 & 84 & $(58-99)$ & - \\
\hline Clinical/other & 29 & 79 & $(21-99)$ & - \\
\hline
\end{tabular}

5-FU=fluorouracil; QHES = Quality of Health Economic Studies.

Mean QHES scores across the levels of evidence graded as high and moderate were 82 for $1 \mathrm{~A}, 93$ for $1 \mathrm{~B}, 71$ for $2 \mathrm{~A}$, and 74 for $2 \mathrm{~B}$. The drug-gene pairs fluoropyrimidine-DPYD for multiple cancers and 6-mercaptopurine-TPMT for acute lymphoblastic leukemia had $1 \mathrm{~A}$ level of evidence, and their respective economic studies had mean QHES scores of 86 and 78 (range $=70-87$ ), respectively. ${ }^{20-22}$ Erlotinib-EGFR and gefitinib-EGFR for lung cancer had 1B level of evidence, and their economic studies had mean QHES scores of 96 (range = 89-99) and 89 (range $=83-95$ ), respectively. ${ }^{23-34}$ The level of evidence for drug-gene pairs in colorectal cancer was 2A for irinotecanUGT1Al and 2B for panitumumab-KRAS and cetuximabRAS/KRAS/BRAF; the mean QHES scores of their economic studies were 71 (range $=25-98), 76$ (range $=72-80)$, and 71 (range $=20-96$ ), respectively. ${ }^{14,35-47}$ The drug-gene pairs cisplatinTPMT/COMT for multiple cancers, carboplatin-BRCA-1-like gene for resistant triple-negative breast cancer, and trastuzumab-HER2 for breast cancer did not have an assessment of strength of association per PharmGKB; the mean QHES scores of the studies assessing these pairs were 58,96 , and 78 (range $=58-89)$, respectively. ${ }^{48-52}$

\section{Economic Evidence Summary}

One hundred nine relevant comparisons were identified within the included studies. These included ICER $(n=58)$ and cost difference $(n=19)$ economic outcomes for pharmacogenetic testing. . $^{4,20-28,30-36,38-51,53}$ Furthermore, comparisons were identified for cetuximab and trastuzumab where pharmacogenetic testing was less effective than the comparator. In these comparisons, all patients in the no-testing cohort received cetuximab or 


\section{Economic Value of Pharmacogenetic Testing for Cancer Drugs with Clinically Relevant Drug-Gene Associations: A Systematic Literature Review}

\section{FIGURE 2 ICERs of Genetic Testing Interventions Based on WTP Thresholds of Geographic Regions}

\begin{tabular}{|c|c|c|c|c|c|}
\hline & All Regions & Canada $(n=6)$ & Asia $(n=12)$ & United States $(n=23)$ & Europe $(n=17)$ \\
\hline \multicolumn{6}{|l|}{$\begin{array}{l}\text { Predictive for response } \\
(\mathrm{n}=53)\end{array}$} \\
\hline $\begin{array}{l}\text { Cetuximab }(n=17) \\
\text { Colorectal cancer }\end{array}$ & & $\mathrm{n}=3$ & $\mathrm{n}=5$ & $\mathrm{n}=2$ & $\mathrm{n}=4$ \\
\hline $\begin{array}{l}\text { Erlotinib }(\mathrm{n}=13) \\
\text { Lung cancer }\end{array}$ & & None available & None available & $\mathrm{n}=10$ & $\mathrm{n}=1$ \\
\hline $\begin{array}{l}\text { Gefitinib }(n=5) \\
\text { Lung cancer }\end{array}$ & & $\mathrm{n}=1$ & $\mathrm{n}=2$ & None available & None available \\
\hline $\begin{array}{l}\text { Trastuzumab }(n=16) \\
\text { Breast cancer }\end{array}$ & & None available & None available & $\mathrm{n}=8$ & $\mathrm{n}=5$ \\
\hline $\begin{array}{l}\text { Panitumumab }(\mathrm{n}=1) \\
\text { Colorectral cancer }\end{array}$ & & $\mathrm{n}=1$ & None available & None available & None available \\
\hline $\begin{array}{l}\text { Carboplatin }(\mathrm{n}=1) \\
\text { Breast cancer }\end{array}$ & & None available & None available & None available & $\mathrm{n}=1$ \\
\hline $\begin{array}{l}\text { Predictive for toxicity } \\
(n=5)\end{array}$ & & None available & None available & & \\
\hline $\begin{array}{l}\text { Irinotecan }(n=4) \\
\text { Colorectal cancer }\end{array}$ & & None available & None available & $\mathrm{n}=1$ & $\mathrm{n}=1$ \\
\hline $\begin{array}{l}\text { 6-Mercaptopurine }(\mathrm{n}=1) \\
\text { Leukemia }\end{array}$ & & None available & None available & None available & $\mathrm{n}=1$ \\
\hline Legend & $\begin{array}{l}\text { Dominant } \\
\text { Not dominant }\end{array}$ & $\begin{array}{l}\text { Dominant } \\
\text { Not dominant } \\
0-20,000 \mathrm{CAD} \\
21,000-80,000 \mathrm{CAD} \\
81,000-150,000 \mathrm{CAD} \\
>150,000 \mathrm{CAD}\end{array}$ & $\begin{array}{l}\text { Dominant } \\
\text { Not dominant } \\
0-20,000 \text { USD } \\
21,000-50,000 \text { USD } \\
51,000-100,000 \text { USD } \\
>100,000 \text { USD }\end{array}$ & $\begin{array}{l}\text { Dominant } \\
\text { Not dominant } \\
0-50,000 \text { USD } \\
51,000-100,000 \text { USD } \\
101,000-150,000 \text { USD } \\
>150,000 \text { USD }\end{array}$ & $\begin{array}{l}\text { Dominant } \\
\text { Not dominant } \\
0-20,000 \text { EUR } \\
21,000-30,000 \text { EUR } \\
31,000-80,000 \text { EUR } \\
>80,000 \text { EUR }\end{array}$ \\
\hline
\end{tabular}

Note: This figure is composed of 2 parts: (1) illustration of ICERs as dominant versus not dominant and (2) illustration of ICERs that are not dominant (i.e., ICER $>0$ ) using WTP threshold categories. Generally, somatic mutation testing was conducted to predict response, whereas germline mutation testing was conducted to predict toxicity. Number of interventions compared, $n=58$; number of studies, $n=25 ; 2$ effect measures included LYs and QALYs; currency year included years 2002 to 2014; study year included years 2004 to 2016.

$C A D=$ Canadian dollar; $E U R=$ euro; ICER=incremental cost-effectiveness ratio; LY=life-year; $2 A L Y=q u a l i t y$-adjusted life-year; USD =U.S. dollar; WTP=willingness-to-pay.

trastuzumab, whereas patients in the pharmacogenetic testing cohort were selected to receive these drugs based on their test results. Wen et al. (2015) reported a comparison where bevacizumab treatment was dominant when compared with cetuximab treatment in the KRAS WT patient population $(n=12){ }^{14,39,40,43,45,51}$ Comparisons were also identified where the outcome of effectiveness was not reported in life-years or quality-adjusted life-years (progression-free survival $[n=1]$, correctly managed cases [ $\mathrm{n}=18]$, adverse event-avoided cases $[n=1]){ }^{29,37,52}$ The number of comparisons found for each drug and in each geographic region varied.

\section{Cost-Effectiveness Studies}

Across all geographic regions and drugs, pharmacogenetic testing compared with an alternative (no testing or less comprehensive testing) was dominant in $21 \%$ of the comparisons 


\section{Economic Value of Pharmacogenetic Testing for Cancer Drugs with Clinically Relevant Drug-Gene Associations: A Systematic Literature Review}

providing ICERs. Predictive testing for response, which is generally testing of somatic mutations, was dominant in 19\% of the comparisons overall and in $42 \%, 21 \%, 17 \%$, and $5 \%$ of the comparisons in Asia, Europe, Canada, and the United States, respectively. Predictive testing for toxicity, which is generally testing of germline mutations, was dominant in $40 \%$ of comparisons overall and in 50\% and 33\% of the comparisons in the United States and Europe, respectively. Only 1 comparison was available for the ICER of pharmacogenetic testing for 6-mercaptopurine, carboplatin, and panitumumab. Comparisons involving irinotecan, gefitinib, trastuzumab, cetuximab, and erlotinib found pharmacogenetic testing to be dominant in $50 \%, 40 \%, 19 \%, 18 \%$, and $15 \%$ of comparisons, respectively. In comparisons where pharmacogenetic testing was not dominant, the ICER values varied across geographic region and drug categories. For example, in the case of erlotinib, $85 \%$ of comparisons were not dominant $(\mathrm{n}=11)$. Of these $11 \mathrm{com}-$ parisons, 10 comparisons were from the United States and 1 comparison was from Europe. There was variability in ICERs across geographic regions because the ICER of the comparison in Europe was in the lowest WTP threshold category, whereas ICERs of comparisons in the United States ranged from the lowest to the highest WTP threshold category. This pattern was also observed for ICER values of cetuximab, gefitinib, and trastuzumab, which demonstrated that there was variability across drugs as well. The case of irinotecan was an exception, for which 1 study in Europe and 1 study in the United States were both in the highest WTP threshold category (Figure 2).

These 58 comparisons were also characterized by 1 of 12 unique treatment strategy combinations. In comparisons of patient selection via pharmacogenetic testing versus no testing, where all patients had access to the drug with relevant drug-gene association, pharmacogenetic testing was dominant in $67 \%$ of comparisons. Likewise, pharmacogenetic testing for patient selection was dominant in comparisons against other patient selection strategies in cases where all patients had access to the drug with a relevant drug-gene association. The proportions of comparisons within WTP threshold categories are illustrated in Figure 3.

\section{Cost Differences and Other Findings}

Eighty-nine percent of the comparisons reporting cost differences were cost saving $(n=17)$. Pharmacogenetic testing was cost saving in studies conducted in the United States $(n=9)$, Europe $(n=6)$, and Asia $(n=1)$. Both cost savings $(n=1)$ and cost increases $(n=2)$ were observed in Canada. Cost increases occurred for TPMT testing to guide 6-mercaptopurine use compared with no testing and enzymatic testing. Fifty-three percent of the comparisons were made between pharmacogenetic testing that guided patient selection and no testing, where all patients had access to the drug with a relevant drug-gene association $(n=10)$. All these comparisons were cost saving.
The remaining cost-saving comparison characteristics were heterogeneous and included comparisons of patient selection using pharmacogenetic testing versus protein expression or clinical features. Pharmacogenetic testing was not consistently dominant or cost saving for comparisons where the outcome of effectiveness was progression-free survival, correctly managed cases, or adverse events avoided.

\section{Discussion}

This systematic literature review summarizes the available evidence for the economic value of pharmacogenetic testing for cancer therapies with clinically relevant drug-gene associations. We also compared the quality of the evidence assessing the economic value of pharmacogenetic testing with the PharmGKB-graded level of evidence for the drug-gene pairs. Based on the findings from this study, we concluded that (a) pharmacogenetic testing is not a universally dominant strategy, and (b) among the comparisons in which pharmacogenetic testing was not a dominant strategy, there was variability in the ICER values, with a significant proportion being in the higher WTP threshold categories. ${ }^{15-17}$ Pharmacogenetic testing was also the less effective intervention in some comparisons with no testing or less comprehensive testing. Furthermore, pharmacogenetic testing was likely to be dominant when specific combinations of treatment strategies were compared. In contrast, pharmacogenetic testing was cost saving in most of the cost-minimization comparisons. These comparisons typically had reduced utilization of the drug of interest with pharmacogenetic testing and, therefore, lower costs. Similar to Hatz et al. (2014), the cost-effectiveness of genetic testing depended on the WTP threshold. ${ }^{54}$

Pharmacogenetic testing is not consistently a dominant strategy for any drug included in this study. Among the included drugs, pharmacogenetic testing is dominant in at most $50 \%$ of the comparisons, and among comparisons in which pharmacogenetic testing is not dominant, the variability in the ICER favors higher WTP threshold categories. Even when geographic regions are accounted for, inconsistencies exist; the only exception is cetuximab in the United States, where the ICERs are consistently high $(>\$ 150,000) \cdot{ }^{14}$ In some cases, the inconsistencies can be attributed to the interventions compared; e.g., for irinotecan in both the United States and Europe, we see high ICERs because the actionable intervention, based on results of pharmacogenetic testing, was the administration of irinotecan along with the high-cost supplemental therapy granulocyte colony-stimulating factor (G-CSF). ${ }^{35,38}$ This granular assessment demonstrates how conclusions regarding cost-effectiveness that are based on a broader perspective can mask important deviations. For example, previous work has indicated that the evidence of cost-effectiveness of genetic testing to guide irinotecan therapy is robust, whereas we found that cost-effectiveness depends on which test-informed 
Patient selection via protein expression or clinical features followed by pharmacogenetic testing and drug with relevant drug-gene association provided VERSUS patient selection via protein expression or clinical features and drug with relevant drug-gene association provided

Patient selection via pharmacogenetic testing and drug with relevant drug-gene association provided VERSUS patient selection via pharmacogenetic testing of fewer genes and drug with relevant drug-gene association provided

Patient selection via pharmacogenetic testing and drug with relevant drug-gene
association provided VERSUS patient selection via pharmacogenetic testing of fewer
genes and drug with relevant drug-gene association provided

Patient selection via pharmacogenetic testing and drug with relevant drug-gene association provided VERSUS patient selection via protein expression or clinical features and pharmacogenetic testing and drug with relevant drug-gene association provided

Patient selection via pharmacogenetic testing and drug with relevant drug-gene association provided VERSUS patient selection via delayed pharmacogenetic testing and chemotherapy, drug without gene-drug association, or best supportive care provided

Patient selection via pharmacogenetic testing and drug with relevant drug-gene association provided VERSUS patient selection via delayed pharmacogenetic testing and drug with relevant drug-gene association provided

Patient selection via pharmacogenetic testing and drug with relevant drug-gene

association provided VERSUS patient selection via pharmacogenetic testing and chemotherapy, drug without gene-drug association, or best supportive care provided

Patient selection via protein expression or clinical features and pharmacogenetic testing and drug with relevant drug-gene association provided VERSUS patient selection not conducted and chemotherapy, drug without gene-drug association, or best supportive care provided

Patient selection via pharmacogenetic testing and drug with relevant drug-gene association provided and additional therapy provided VERSUS patient selection via protein expression or clinical features and drug with relevant drug-gene association provided

Patient selection via pharmacogenetic testing and drug with relevant drug-gene association provided and additional therapy provided VERSUS patient selection not conducted and drug with relevant drug-gene association provided 


\section{Economic Value of Pharmacogenetic Testing for Cancer Drugs with Clinically Relevant Drug-Gene Associations: A Systematic Literature Review}

actionable intervention is selected (i.e., dose reduction of irinotecan versus addition of high-cost supplemental therapy G-CSF). ${ }^{35,55}$ It should be noted that for irinotecan, 6-mercaptopurine, carboplatin, gefitinib, and panitumumab, cost-effectiveness data are limited. More data are needed to understand the nuances related to the economic value of pharmacogenetic testing with respect to these (and other) drugs.

Considering that the WTP threshold is often higher for cancer drugs than noncancer drugs, it is notable that the majority of comparisons containing erlotinib as the gene (EGFR)relevant drug had ICERs at least within the second-lowest WTP threshold category (i.e., <80,000 Canadian dollars in Canada; $<50,000$ U.S. dollars in Asia; <100,000 U.S. dollars in the United States; $<30,000$ euros in Europe) for each geographic region; however, the majority of comparisons for cetuximab and trastuzumab as gene (EGFR and HER-2)-relevant drugs had ICERs above this WTP threshold. ${ }^{56}$ Given that treatment approaches in oncology are heterogeneous and dynamic due to the nature of the diseases and that use of pharmacogenetic tests is reasonable at multiple points of treatment, variability of comparisons within cost-effectiveness analyses are expected; such variabilities can potentially affect the relative cost-effectiveness of pharmacogenetic testing. ${ }^{57,58}$

Past reviews focused on the main findings of studies and concluded that genetic testing is cost-effective in more cases than not. Our conclusion is not consistent with this, which in part may be due to methodological differences. We identified and accounted for every relevant comparison with pharmacogenetic testing as permitted from the data reported in each publication. This method of reporting provides the capability to thoroughly report all relevant data from each study, thus reducing the chance of missing negative or neutral findings. For example, in studies assessing cost-effectiveness of cetuximab, the overall study findings conclude that pharmacogenetic testing is dominant when both interventions include cetuximab; however, if pharmacogenetic testing-guided cetuximab treatment is compared against best supportive care, the ICER is no longer dominant. ${ }^{40,46}$ Since we assessed the literature at a comparison level rather than a study level, we were able to report all ICERs from the included studies, including those that the authors did not explicitly summarize.

The inconsistencies of ICERs align with findings by D'Andrea et al. (2015), where 138 identified ICERs ranged from "costsaving" to "> 78,000 euros" to "higher cost and less effective." Similar findings are reported in other reviews on the topic of cost-effectiveness of personalized medicine. ${ }^{8,54,60}$ Furthermore, no meta-analysis was identified on this general topic as each of the previous review articles note that the included studies had a high degree of heterogeneity and, therefore, results could not be pooled across studies. ${ }^{8,54,55,59,61}$ Thus, qualitative synthesis is the chosen method of analysis. ${ }^{55,61}$ Our method of reporting the findings differed, however, since we decided against using narrative synthesis and provided pictorial representations of the extracted data. To our knowledge, this is the first systematic literature review focused on understanding the economic value of cancer drugs with relevant drug-gene pair associations. Furthermore, in addition to having this narrower focus, the present study used a search term that included the names of the drugs and genes of interest, in addition to general terms relating to pharmacoeconomics and precision medicine. This arrangement enabled us to comprehensively explore the published body of literature and reduce the probability of missing any relevant articles. A similar strategy was adopted in past reviews where the authors focused only on specific drugs pertinent to their research question. ${ }^{8,62}$ While our study contains a heterogenous sample of comparisons, our narrow focus on oncology enabled us to draw conclusions that are specific to 1 disease area. Furthermore, we highlight heterogeneity that may have been masked in past reviews because our methods reduce the chances of high-cost diseases being averaged out by other lower-cost diseases. Lastly, narrowing the focus to oncology enabled us to find that the majority of economic analyses of pharmacogenetic testing are for somatic mutation testing to predict response. This provides insights for the direction of future research.

Our quality assessment aligned with findings from previous reviews focused on precision medicine that used the QHES tool. ${ }^{59,61,63}$ The QHES is a quick and relatively easy way to identify high-quality studies and has found ready application in the field. ${ }^{12,64-66}$ We reported the QHES score for 2 reasons: as a way to characterize the quality of the articles and to provide a benchmark for the quality of economic evaluations of pharmacogenetic testing. On average, the articles included in this study were good-quality studies, with a mean (standard deviation) QHES score of 80 (22) and ICC of 0.82 (95\% CI =0.46-0.93). This ICC point estimate exceeds the threshold of 0.8 , which has been suggested as the threshold for excellent agreement between raters. ${ }^{67}$ The $95 \%$ CI on the ICC is consistent with intervals seen in previous studies. Ofman et al. (2003) suggested using a minimum QHES score of 75 to identify a good-quality economic evaluation. ${ }^{12}$ Using this threshold, $77 \%(n=27)$ of the included studies were considered good-quality economic evaluations. Similar to findings from previous work, the articles receiving a lower QHES score in the current study generally lacked information regarding the study perspective, analytic method, sensitivity analysis, or potential sources of bias. ${ }^{66}$

In our study, 1 of 5 papers did not report an analytic approach. Incompleteness in reporting technical details of modeling studies has been the subject of best practice reports. These reports provide guidance that will be relevant for future studies on the value of pharmacogenetic testing. The findings of this study provide the following insights for the design and conduct of future work: 


\section{Economic Value of Pharmacogenetic Testing for Cancer Drugs with Clinically Relevant Drug-Gene Associations: A Systematic Literature Review}

1. Future economic studies on pharmacogenetic testing should follow existing guidelines for the design, conduct, and reporting of modeling studies and cost-effectiveness studies. ${ }^{68}$ Relevant stakeholders should be consulted so that the interventions compared represent real-world use of drugs and technologies. Furthermore, sensitivity analyses should be conducted because they broaden the generalizability of results and make findings more useful for decision makers. ${ }^{69}$ Among the studies on this topic, differences are expected regarding populations, interventions compared, perspectives, and clinical settings, depending on the research question being asked. Therefore, it is critical to report the results in their most granular form (i.e., cost and effect of each intervention, in addition to the calculated ICER with a corresponding 95\% CI).

2. Future reviews evaluating the economics of precision medicine should provide rationale for the scope of coverage (e.g., therapeutic areas included) and granularity of data extraction in the study design. Our study demonstrates that including 1 therapeutic area and extracting data at a comparison level from included studies has implications for the conclusion reached.

\section{Limitations}

This study has some limitations to consider. First, only a qualitative analysis was performed due to significant heterogeneity in the articles included. Thus, the results only provide a highlevel representation of the economic value of pharmacogenetic testing for cancer drugs.

Second, this study only included cancer drugs from the PharmGKB Clinical Annotations list, which is not a comprehensive list of cancer drugs where pharmacogenetic testing is relevant. Furthermore, we accessed this list on October 1, 2016. Therefore, we are only able to provide a broad overview on the subject of the economic value of pharmacogenetic testing in clinical oncology using published literature between 2000 and 2016.

Finally, quality assessment was conducted using the QHES tool, which is a subjective tool that lacks flexibility to award partial credit in situations of nuanced technical differences between articles (e.g., missing 1 component of a question with multiple components). This may have contributed to the variability in scores across articles and between authors. ${ }^{12,63}$

\section{Conclusions}

The economic value of pharmacogenetic testing for cancer drugs with clinically relevant drug-gene associations is unclear-a limited number of studies provided evidence that pharmacogenetic testing is a dominant strategy. Among those that are not dominant, there is significant variability in ICERs across comparisons. The majority of cost-minimization studies are cost saving. The current review yielded recommendations for conducting economic evaluations of pharmacogenetic testing and for developing reviews of published economic evaluation studies. While future studies may continue the current emphasis on somatic mutation testing to predict efficacy, there should be greater adherence to available best practice guidelines for conducting and reporting economic evaluation studies. With regard to developing reviews of economic evaluations, we recommend focusing on 1 therapeutic area and reporting findings at the comparison level. With these recommendations in mind, future research can provide evidence to clarify the value of pharmacogenetic testing.

\section{Authors}

FAHIM FARUQUE, PharmD; HEEJUNG NOH, PharmD; and EDWARD NEUBERGER, PharmD, MBA, University of Maryland School of Pharmacy, Baltimore. ARIF HUSSAIN, MD, Baltimore VA Medical Center and University of Maryland Greenebaum Comprehensive Cancer Center, Baltimore. EBERECHUKWU ONUKWUGHA, MS, PhD, Department of Pharmaceutical Health Services Research, University of Maryland School of Pharmacy, Baltimore.

AUTHOR CORRESPONDENCE: Fahim Faruque, PharmD, University of Maryland School of Pharmacy, 20 N. Pine St., Baltimore, MD 21201. Tel.: 443.676.7088; E mail: ffaruque@umaryland.edu.

\section{DISCLOSURES}

No outside funding supported this review. Part of Hussain's research time was supported by a Merit Review Award (I01 BX000545), Medical Research Service, U.S. Department of Veterans Affairs. Hussain also reports personal fees from Bristol-Myers Squibb, AstraZeneca, Novartis, Bayer HealthCare Pharmaceuticals, and France Foundation, outside the submitted work. Onukwugha reports grants from Pfizer and Bayer HealthCare Pharmaceuticals, along with advisory board fees from Novo Nordisk, outside the submitted work. Faruque, Neuberger, and Noh have nothing to disclose.

\section{REFERENCES}

1. International Council for Harmonisation of Technical Requirements for Pharmaceuticals for Human Use. E15, Definitions in pharmacogenomics, pharmacogenetics. Available at: http://www.ich.org/products/guidelines/efficacy/efficacy-single/article/definitions-for-genomic-biomarkers-pharmacogenomics-pharmacogenetics-genomic-data-and-sample-cod.html. Accessed November 28, 2018.

2. Reed M, Page M. Recognizing the value of precision medicine: oncology and beyond. Am J Manag Care. Supplement. August 24, 2017. Available at: https://www.ajmc.com/journals/supplement/2017/exploring-the-evolvinglandscape-of-precision-medicine/recognizing-the-value-of-precision-medicine-oncology-and-beyond-article. Accessed November 28, 2018.

3. National Cancer Institute. Precision medicine in cancer treatment. 2017. Available at: https://www.cancer.gov/about-cancer/treatment/types/precisionmedicine\#1. Accessed November 28, 2018.

4. PharmGKB. Level $1 \mathrm{~A} \& 1 \mathrm{~B}$ clinical annotations. Available at: https:// www.pharmgkb.org/. Accessed November 28, 2018. 


\section{Economic Value of Pharmacogenetic Testing for Cancer Drugs with Clinically Relevant Drug-Gene Associations: A Systematic Literature Review}

5. Kamel HFM, Al-Amodi HSAB. Exploitation of gene expression and cancer biomarkers in paving the path to era of personalized medicine. Genomics Proteomics Bioinformatics. 2017;15(4):220-35.

6. Cancer Treatment Centers of America. FDA approves first cancer treatment based on genetic makeup, not tumor location. 2017. Available at: https://www.cancercenter.com/discussions/blog/fda-approves-first-cancer-treatment-based-on-genetic-makeup-not-tumor-location/. Accessed November 28, 2018

7. U.S. Food and Drug Administration. FDA grants accelerated approval to pembrolizumab for first tissue/site agnostic indication. 2017. Available at: https://www.fda.gov/Drugs/InformationOnDrugs/ApprovedDrugs/ ucm560040.htm. Accessed November 28, 2018.

8. Verbelen M, Weale ME, Lewis CM. Cost-effectiveness of pharmacogeneticguided treatment: are we there yet? Pharmacogenomics J. 2017;17(5):395-402.

9. Faruque F, Onukwugha E. A review of the economic value of genetic testing for cancer therapies with clinically relevant variant-drug associations. Poster presented at: Academy of Managed Care Pharmacy 2015 Nexus; October 27-28, 2015; Orlando, FL.

10. Liberati A, Altman DG, Tetzlaff J, et al. The PRISMA statement for reporting systematic reviews and meta-analyses of studies that evaluate health care interventions: explanation and elaboration. J Clin Epidemiol. 2009;62(10):el-34

11. Faruque F, Noh H, Onukwugha E. Economic value of genetic testing for cancer therapies with clinically relevant drug-gene associations: a systematic literature review. 2017. Available at: http://www.crd.york.ac.uk/PROSPERO/ display_record.php?ID=CRD42016050020. Accessed November 28, 2018.

12. Ofman JJ, Sullivan SD, Neumann PJ, et al. Examining the value and quality of health economic analyses: implications of utilizing the QHES. J Manag Care Pharm. 2003;9(1):53-61. Available at: https://www.jmcp.org/ doi/10.18553/jmcp.2003.9.1.53.

13. Limone BL, Baker WL, Kluger J, Coleman CI. Novel anticoagulants for stroke prevention in atrial fibrillation: a systematic review of cost-effectiveness models. PLoS One. 2013;8(4):e62183.

14. Behl AS, Goddard KAB, Flottemesch TJ, et al. Cost-effectiveness analysis of screening for KRAS and BRAF mutations in metastatic colorectal cancer. J Natl Cancer Inst. 2012;104(23):1785-95.

15. Schwarzer R, Rochau U, Saverno K, et al. Systematic overview of costeffectiveness thresholds in ten countries across four continents. J Comp Eff Res. 2015;4(5):485-504.

16. Tengs TO. Cost-effectiveness versus cost-utility analysis of interventions for cancer: does adjusting for health-related quality of life really matter? Value Health. 2004;7(1):70-78.

17. Cleemput I, Neyt M, Thiry N, De Laet C, Leys M. Threshold values for cost-effectiveness in health care. Health technology assessment (HTA). KCE reports 100C (D/2008/10.273/96). Brussels: Belgian Health Care Knowledge Centre; 2008. Available at: https://kce.fgov.be/sites/default/files/atoms/files/ d20081027396.pdf. Accessed December 27, 2018.

18. Kulesher R, Forrestal E. International models of health systems financing. J Hosp Adm. 2014;3(4):127-39.

19. InterNations. Healthcare and education in Mexico. Available at: https:// www.internations.org/mexico-expats/guide/living-in-mexico-15386/healthcare-and-education-in-mexico-2. Accessed November 28, 2018.

20. Deenen MJ, Meulendijks D, Cats A, et al. Upfront genotyping of DPYD2A to individualize fluoropyrimidine therapy: a safety and cost analysis. J Clin Oncol. 2016;34(3):227-34.

21. Donnan JR, Ungar WJ, Mathews M, Hancock-Howard RL, Rahman P. A cost effectiveness analysis of thiopurine methyltransferase testing for guiding 6-mercaptopurine dosing in children with acute lymphoblastic leukemia. Pediatr Blood Cancer. 2011;57(2):231-39.
22. Van Den Akker-Van Marle ME, Gurwitz D, Detmar SB, et al. Costeffectiveness of pharmacogenomics in clinical practice: a case study of thiopurine methyltransferase genotyping in acute lymphoblastic leukemia in Europe. Pharmacogenomics. 2006;7(5):783-92.

23. Lim EA, Lee H, Bae E, Lim J, Shin YK, Choi S. Economic evaluation of companion diagnostic testing for EGFR mutations and first-line targeted therapy in advanced non-small cell lung cancer patients in South Korea. PLOS ONE. 2016;11(8):e0160155.

24. Schremser K, Rogowski WH, Adler-Reichel S, Tufman ALH, Huber RM, Stollenwerk B. Cost-effectiveness of an individualized first-line treatment strategy offering erlotinib based on EGFR mutation testing in advanced lung adenocarcinoma patients in Germany. Pharmacoeconomics. 2015;33(11):1215-28.

25. Romanus D, Cardarella S, Cutler D, Landrum MB, Lindeman NI, Gazelle GS. Cost-effectiveness of multiplexed predictive biomarker screening in nonsmall-cell lung cancer. J Thorac Oncol. 2015;10(4):586-94.

26. Handorf EA, McElligott S, Vachani A, et al. Cost effectiveness of personalized therapy for first-line treatment of stage IV and recurrent incurable adenocarcinoma of the lung. J Oncol Pract. 2012;8(5):267-74.

27. Borget I, Cadranel J, Pignon J, et al. Cost-effectiveness of three strategies for second-line erlotinib initiation in nonsmall-cell lung cancer: the ERMETIC study part 3. Eur Respir J. 2012;39(1):172-79.

28. Carlson JJ, Garrison LP, Ramsey SD, Veenstra DL. The potential clinical and economic outcomes of pharmacogenomic approaches to EGFRtyrosine kinase inhibitor therapy in non-small-cell lung cancer. Value Health. 2009;12(1):20-27.

29. Arrieta O, Anaya P, Morales-Oyarvide V, Ramírez-Tirado LA, Polanco AC. Cost-effectiveness analysis of EGFR mutation testing in patients with nonsmall cell lung cancer (NSCLC) with gefitinib or carboplatin-paclitaxel. Eur J Health Econ. 2016;17(7):855-63.

30. Narita Y, Matsushima Y, Shiroiwa T, et al. Cost-effectiveness analysis of EGFR mutation testing and gefitinib as first-line therapy for non-small cell lung cancer. Lung Cancer. 2015;90(1):71-77.

31. Permsuwan U, Niamhun N, Tanatip N, Thongprasert S. Epidermal growth factor receptor mutation testing in Thailand: a cost-utility analysis. Value Health Reg Issues. 2014;3(1):39-43.

32. Zhu J, Li T, Wang X, et al. Gene-guided gefitinib switch maintenance therapy for patients with advanced EGFR mutation-positive non-small cell lung cancer: an economic analysis. BMC Cancer. 2013;13:39.

33. De Lima Lopes G, Segel JE, Tan DSW, Do YK, Mok T, Finkelstein EA. Cost-effectiveness of epidermal growth factor receptor mutation testing and first-line treatment with gefitinib for patients with advanced adenocarcinoma of the lung. Cancer. 2012;118(4):1032-39.

34. Health Quality Ontario. Epidermal growth factor receptor mutation (EGFR) testing for prediction of response to EGFR-targeting tyrosine kinase inhibitor (TKI) drugs in patients with advanced non-small-cell lung cancer: an evidence-based analysis. Ont Health Technol Assess Ser. 2010;10(24):1-48.

35. Butzke B, Oduncu FS, Severin F, et al. The cost-effectiveness of UGTlAl genotyping before colorectal cancer treatment with irinotecan from the perspective of the German statutory health insurance. Acta Oncol. 2016;55(3):318-28.

36. Saab YB, Taimourlangaee, Zeenny R. Effect of the global variation of the genetic biomarker uridine diphosphate glucuronosyl transferase. Int $J$ Pharmcy Pharm Sci. 2014;6(6):632-37.

37. Pichereau S, Le Louarn A, Lecomte T, Blasco H, Le Guellec C, Bourgoin H. Cost-effectiveness of UGT1Al*28 genotyping in preventing severe neutropenia following FOLFIRI therapy in colorectal cancer. J Pharm Pharm Sci. 2010;13(4):615-25.

38. Obradovic M, Mrhar A, Kos M. Cost-effectiveness of UGTlAl genotyping in second-line, high-dose, once every 3 weeks irinotecan monotherapy treatment of colorectal cancer. Pharmacogenomics. 2008;9(5):539-49. 


\section{Economic Value of Pharmacogenetic Testing for Cancer Drugs with Clinically Relevant Drug-Gene Associations: A Systematic Literature Review}

39. Vijayaraghavan A, Efrusy MB, Göke B, Kirchner T, Santas CC, Goldberg RM. Cost-effectiveness of KRAS testing in metastatic colorectal cancer patients in the united states and Germany. Int J Cancer. 2012;131(2):438-45.

40. Health Quality Ontario. KRAS testing for anti-EGFR therapy in advanced colorectal cancer: an evidence-based and economic analysis. Ont Health Technol Assess Ser. 2010;10(25):1-49.

41. Zhou J, Zhao R, Wen F, et al. Economic evaluation study (CHEERcompliant): cost-effectiveness analysis of RAS screening for treatment of metastatic colorectal cancer based on the CALGB 80405 trial. Medicine. 2016;95(27).

42. Kircher SM, Mohindra N, Nimeiri H. Cost estimates and economic implications of expanded RAS testing in metastatic colorectal cancer. Oncologist. 2015;20(1):14-18

43. Wen F, Yang Y, Zhang P, et al. Cost-effectiveness of RAS screening before monoclonal antibodies therapy in metastatic colorectal cancer based on FIRE3 study. Cancer Biol Ther. 2015;16(11):1577-84.

44. Barone C, Pinto C, Normanno N, et al. KRAS early testing: consensus initiative and cost-effectiveness evaluation for metastatic colorectal patients in an Italian setting. PLoS One. 2014;9(1):e85897.

45. Blank PR, Moch H, Szucs TD, Schwenkglenks M. KRAS and BRAF mutation analysis in metastatic colorectal cancer: a cost-effectiveness analysis from a swiss perspective. Clin Cancer Res. 2011;17(19):6338-46.

46. Shiroiwa T, Motoo Y, Tsutani K. Cost-effectiveness analysis of KRAS testing and cetuximab as last-line therapy for colorectal cancer. Mol Diagn Ther. 2010;14(6):375-84.

47. Mittmann N, Au HJ, Tu D, et al. Prospective cost-effectiveness analysis of cetuximab in metastatic colorectal cancer: evaluation of National Cancer Institute of Canada clinical trials group CO.17 trial. J Natl Cancer Inst. 2009;101(17):1182-92

48. Dionne F, Mitton C, Rassekh R, et al. Economic impact of a genetic test for cisplatin-induced ototoxicity. Pharmacogenomics J. 2012;12(3):205-13.

49. Miquel-Cases A, Steuten LMG, Retèl VP, van Harten WH. Early stage cost-effectiveness analysis of a BRCAl-like test to detect triple negative breast cancers responsive to high dose alkylating chemotherapy. Breast. 2015;24(4):397-405.

50. Lidgren M, Jönsson B, Rehnberg C, Willking N, Bergh J. Costeffectiveness of HER2 testing and 1-year adjuvant trastuzumab therapy for early breast cancer. Ann Oncol. 2008;19(3):487-95

51. Elkin EB, Weinstein MC, Winer EP, Kuntz KM, Schnitt SJ, Weeks JC. HER-2 testing and trastuzumab therapy for metastatic breast cancer: a costeffectiveness analysis. J Clin Oncol. 2004;22(5):854-63.

52. Morelle M, Hasle E, Treilleux I, et al. Cost-effectiveness analysis of strategies for HER2 testing of breast cancer patients in France. Int J Technol Assess Health Care. 2006;22(3):396-401.

53. Königsberg R, Hulla W, Klimpfinger M, et al. Clinical and economic aspects of KRAS mutational status as predictor for epidermal growth factor receptor inhibitor therapy in metastatic colorectal cancer patients. Oncology (Switzerland). 2011;81(5-6):359-64.
54. Hatz MH, Schremser K, Rogowski WH. Is individualized medicine more cost-effective? A systematic review. Pharmacoeconomics. 2014;32(5):443-55.

55. Plumpton CO, Roberts D, Pirmohamed M, Hughes DA. A systematic review of economic evaluations of pharmacogenetic testing for prevention of adverse drug reactions. Pharmacoeconomics. 2016;34(8):771-93.

56. Bae YH, Mullins CD. Do value thresholds for oncology drugs differ from nononcology drugs? J Manag Care Spec Pharm. 2014;20(11):1086-92. Available at: https://www.jmcp.org/doi/10.18553/jmcp.2014.20.11.1086.

57. Payne K, Shabaruddin FH. Cost-effectiveness analysis in pharmacogenomics. Pharmacogenomics. 2010;11(5):643-46.

58. Shabaruddin FH, Fleeman ND, Payne K. Economic evaluations of personalized medicine: existing challenges and current developments. Pharmgenomics Pers Med. 2015;8:115-26.

59. D'Andrea E, Marzuillo C, Pelone F, De Vito C, Villari P. Genetic testing and economic evaluations: a systematic review of the literature. Epidemiol Prev. 2015;39(4 Suppl 1):45-50.

60. Phillips KA, Ann Sakowski J, Trosman J, Douglas MP, Liang SY, Neumann P. The economic value of personalized medicine tests: what we know and what we need to know. Genet Med. 2014;16(3):251-57.

61. Plothner M, Ribbentrop D, Hartman JP, Frank M. Cost-effectiveness of pharmacogenomic and pharmacogenetic test-guided personalized therapies: a systematic review of the approved active substances for personalized medicine in Germany. Adv Ther. 2016;33(9):1461-80.

62. Beaulieu M, de Denus S, Lachaine J. Systematic review of pharmacoeconomic studies of pharmacogenomic tests. Pharmacogenomics. 2010;11(11):1573-90.

63. Wong WB, Carlson JJ, Thariani R, Veenstra DL. Cost effectiveness of pharmacogenomics: a critical and systematic review. Pharmacoeconomics. 2010;28(11):1001-13

64. Au F, Prahardhi S, Shiell A. Reliability of two instruments for critical assessment of economic evaluations. Value Health. 2008;11(3):435-39.

65. Gerkens S, Crott R, Cleemput I, et al. Comparison of three instruments assessing the quality of economic evaluations: a practical exercise on economic evaluations of the surgical treatment of obesity. Int J Technol Assess Health Care. 2008;24(3):318-25.

66. Tran BX, Nong VM, Maher RM, Nguyen PK, Luu HN. A systematic review of scope and quality of health economic evaluation studies in Vietnam. PLoS One. 2014;9(8):e103825.

67. Landis JR, Koch GG. The measurement of observer agreement for categorical data. Biometrics. 1977;33(1):159-74.

68. Caro JJ, Briggs AH, Siebert U, Kuntz KM; ISPOR-SMDM Modeling Good Research Practices Task Force. Modeling good research practices-overview: a report of the ISPOR-SMDM modeling good research practices task force-1. Value Health. 2012;15(6):796-803.

69. Jaime Caro J, Eddy DM, Kan H, et al. Questionnaire to assess relevance and credibility of modeling studies for informing health care decision making: an ISPOR-AMCP-NPC good practice task force report. Value Health. 2014;17(2):174-82. 


\section{Economic Value of Pharmacogenetic Testing for Cancer Drugs with Clinically \\ Relevant Drug-Gene Associations: A Systematic Literature Review}

\section{APPENDIX Review Methods}

\section{Search Strategy}

\section{Query}

\#4 \#1 AND \#2 AND \#3 AND [humans]/lim AND [english]/lim AND [abstracts]/lim AND [1-1-2000]/sd NOT [15-9-2016]/sd

\#3 anthracycline OR daunorubicin OR doxorubicin OR idarubicin OR mitoxantrone OR valrubicin OR capecitabine OR carboplatin OR cetuximab OR cisplatin OR cyclophosphamide OR docetaxel OR epirubicin OR erlotinib OR etoposide OR fluorouracil OR gefitinib OR gemcitabine OR irinotecan OR leucovorin OR mercaptopurine OR methotrexate OR oxaliplatin OR paclitaxel OR radiotherapy OR rituximab OR sunitinib OR tamoxifen OR tegafur OR thioguanine OR trastuzumab OR lapatinib OR panitumumab OR fludarabine OR cladribine OR clofarabine OR nelarabine OR pentostatin OR azacitidine OR cytarabine OR decitabine OR purine OR pyrimidine 'gene mutation'/exp OR 'genetics'/exp OR 'genetic screening'/exp OR 'single nucleotide polymorphism'/exp OR 'sequence analysis'/ exp OR 'personalized medicine'/exp OR 'pharmacogenomics'/exp OR (gene OR genes OR genetic) NEAR/3 (test OR tests OR tested OR testing) OR genomic* OR dna NEAR/3 sequenc* OR pharmacogen* OR (individualized OR individualised OR personalized OR personalised OR precision) NEAR/3 medicine OR single NEAR/3 nucleotide NEAR/3 polymorphism* OR snp OR dpyd OR tpmt OR egfr OR xpc OR mthfr OR gstpl OR cyp2d6 OR tancl OR ugtlal OR nqol OR slcolbl OR tyms OR abcbl OR fcgr3a OR erccl OR sod2 OR cbr3 OR ugtla9 OR c8orf34 OR sema3c OR fcgr2a OR egf OR nt5c2 OR xrccl OR dync2hl OR gstml OR kras OR umps OR fastkd3 OR tp53 OR slc28a3 OR mtrr OR has3 OR hla-dqal OR nudt15 (economic AND evaluation*) OR (economic AND outcome*) OR (analy* NEAR/3 (cost OR costs OR economic*)) OR (cost NEAR/3 (effective* OR benefit OR utility)) OR 'cost of illness'

\section{Drug List and Drug Selection}

PharmGKB accessed on October 1, 2016. The following cancer treatment-related drug terms were identified through the PharmGKB Clinical Annotations list: Alkylating agents, anthracyclines and related substances, antineoplastic agents, azathioprine, capecitabine, carboplatin, cetuximab, cisplatin, cyclophosphamide, docetaxel, epirubicin, erlotinib, etoposide, fluorouracil, gefitinib, gemcitabine, irinotecan, lapatinib, leucovorin, mercaptopurine, methotrexate, oxaliplatin, paclitaxel, panitumumab, platinum, platinum compounds, purine analogs, pyrimidine analogs, radiotherapy, rituximab, SN-38, sunitinib, tamoxifen, tegafur, thioguanine, trastuzumab.

The following cancer drug terms were included in the search term: Anthracycline, daunorubicin, doxorubicin, idarubicin, mitoxantrone, valrubicin, capecitabine, carboplatin, cetuximab, cisplatin, cyclophosphamide, docetaxel, epirubicin, erlotinib, etoposide, fluorouracil, gefitinib, gemcitabine, irinotecan, leucovorin, mercaptopurine, methotrexate, oxaliplatin, paclitaxel, radiotherapy, rituximab, sunitinib, tamoxifen, tegafur, thioguanine, trastuzumab, lapatinib, panitumumab, fludarabine, cladribine, clofarabine, nelarabine, pentostatin, azacitidine, cytarabine, decitabine, purine, pyrimidine.

When drug classes were listed, all drugs within that class were included as categorized within PharmGKB. The only time this was not done was for antineoplastic agents and alkylating agents because they are a broad class description. The PharmGKB Clinical Annotations page was checked for antineoplastic agents with respect to gene TP53 mutations, and relevant agents are on the included drug list: Cisplatin, cyclophosphamide, fluorouracil, paclitaxel.

PharmGKB Clinical Annotations page was checked for alkylating agents with respect to gene NQO1 mutations and the relevant agent is on the list: cyclophosphamide.

PharmGKB lists the following as anthracyclines: Daunorubicin, doxorubicin, idarubicin, mitoxantrone, valrubicin.

PharmGKB lists the following as purine analogs: Azathioprine, cladribine, clofarabine, fludarabine, mercaptopurine, nelarabine, thioguanine, pentostatin. Azathioprine is not indicated for cancer treatment and therefore excluded from the list.

PharmGKB lists the following as pyrimidine analogs: Azacitidine, capecitabine, cytarabine, decitabine, fluorouracil, gemcitabine, tegafur.

PharmGKB lists the following as platinum compounds: Oxaliplatin, carboplatin, cisplatin.

Platinum and platinum compounds were not included in the search as only 3 drugs are in this class and inclusion of these terms is redundant. SN-38 is excluded because it is the active metabolite of irinotecan and inclusion would be redundant. 